\title{
Contraction Mapping Principle Approach to Differential Equations
}

\author{
Bishnu P. Dhungana \\ Department of Mathematics, Mahendra Ratna Campus \\ Tribhuvan University, Kathmandu Nepal
}

\begin{abstract}
Using an extension of the contraction mapping principle, a new approach has been proposed in proving the existence of unique solutions of some differential equations.
\end{abstract}

Key words: contraction, existence and uniqueness, differential equations.

\section{Introduction}

Over the past few decades there has been a clear emergence of the idea of contraction mapping inthe realm of nonlinear functional analysis. With a germ of this idea, it was Banach who was first able to introduce and prove a very powerful principle, called Contraction Mapping Principle.

Beginning from the idea of a contraction $T$ and its fixed point (Baily 1966, Rubinstein 1998, Yosida 1978) we state the principle and extend it to iterates i.e. a result similar to contraction mapping principle is obtained for a mapping $T$ (not contraction) provided that some iterate of is a contraction. This paper is mainly concemed with the extraordinary applicability and effectiveness of the principle to evolve a number of useful results in differential equations. Particularly the strength will be given for a new approach in proving the existence of unique solutions of some differential equations with some initial conditions. Compared to the proofs through Picard iterates (Braun 1993) the method of our proof based on an extension of contraction mapping principle is a new approach. In fact the major technical novelty is to obtain the result (3.9) proving that is a contraction for some positive integer. Finally, some examples to illustrate the results are suitably provided.

\section{Preliminaries}

Many equations which are of interest in applications can be put in the form 0where is a mapping of some subset of a metric space into itself. Such a pointquite naturally is called a fixed point of . An ancient method of solving equations of the fom is the method of iteration i.e. an initial approximationis chosen and successive approximationsare generated by the formula

$$
x_{n}=T\left(x_{n-1}\right) \quad(n=1,2, \cdots)
$$

If the mappingis continuous and if the sequence $\left(x_{n}\right)$ converges to $w$, then

$$
w=\lim _{n \rightarrow \infty} x_{n}=\lim _{n \rightarrow \infty} T\left(x_{n-1}\right)=T\left(\lim _{n \rightarrow \infty} x_{n-1}\right)=T(w)
$$

Thus for continuous mapping $T$ if the process (2.1) converges at all, then it converges to a fixed point of $T$. However to prove the convergence of (2.1), we will in general need a condition on $T$ which is much stronger than continuity. Specific ally, we will require that $T$ be contraction in the sense that it always maps any two points closer together in uniform way as expressed by the following definition.

Definition 2.1: Let $(X, d)$ be a metric space. Then a contraction of $(X, d)$ is a mapping $T: X \rightarrow X$ with the property that for some re al number $k<1$, $d(T(x), T(y)) \leq k d(x, y) \quad \forall x, y \in X$.

Note that a differentiable mapping $T:[a, b] \rightarrow[a, b]$ is a contraction if and only if there is a mumber $k<1$ with $\left|T^{\prime}(x)\right| \leq k \quad \forall x, y \in(a, b)$. 
Nepal Journal of Science and Technology 10 (2009) 149-153

Theorem 2.1: (Contraction Mapping Principle, [2]) Let $T: X \rightarrow X$ be a contraction of a complete metric space $(X, d)$. Then $T$ has a unique fixed point i.e. there exists a unique point $w$ in $X$ such that $T(w)=w$. Furthermore, if $x_{0}$ is any point of $X$ and $\left(x_{n}\right)$ is a sequence of iterates defined by $x_{n}=T\left(x_{n-1}\right), n=1,2, \cdots$. Theno $\lim _{n \rightarrow \infty} x_{n}=w$.

Remark 2.1: Both conditions of Theorem 1.1 are necessary sincethe mapping $T:(0,1] \rightarrow(0,1]$ defined by $T(x)=x / 2$ is a contraction map but has no fixed point since $(0,1]$ is not a complete metric space. the mapping $T: R \rightarrow R$ defined by $T(x)=x+5$ is not a contraction and has no fixed point although $R$ is complete.

If $T$ is a contraction mapping, then $T^{n}$ where $n$ is a positive integer, is clearly a contraction mapping. However the converse may not be true as can be seen from the following example.

Example 2.1: The function $T: R \rightarrow R$ defined by $T(x)=e^{-x}$ is not a contraction, but that $T^{2}$ is.

Thus we see that provided some iterate of $T$ is a contraction we still get a fixedpoint result similar to the contraction mapping principle for $T$. The following theorem is an extension of the principle to iterates.

Theorem 2.2: Let $(X, d)$ be a complete metric space and let $T: X \rightarrow X$ have the property that for some integer $N>0$, the iterate $T^{N}$ is a contraction of $X$. Then Thas a unique fixed point ie there exists a unique point $w$ in $X$ such that $T(w)=w$. Furthemore, if $x_{1}$ is in $X$, then the sequence of iterates defined by $x_{n+1}=T\left(x_{n}\right), n \geq 1$ converges to $w$.

Proof: Since $T^{N}$ is a contraction of the complete metric space $(X, d)$, it has a unique fixed point $w$, say. It then follows that
$T(w)=T\left(T^{N}(w)\right)=T^{N+1}(w)=T^{N}(T(w))$.

This shows that $T(w)$ is another fixed point of $T^{N}$. From uniqueness, $T$ has a fixed point $w$ in $X$ and moreover it is unique since any point which $T$ fixes clearly remains fixed by $T^{N}$. Finally to see that $x_{1}, x_{2}, x_{3}, \cdots$ converges to $w$, we re-label $T^{N}$ as $g$ and note that

$x_{N+1}=T\left(x_{N}\right)=T\left(T\left(x_{N-1}\right)\right)=\cdots=T^{N}\left(T\left(x_{1}\right)\right)=g\left(x_{1}\right)$
$x_{N+2}=T\left(x_{N+1}\right)=T\left(T\left(x_{N}\right)\right)=\cdots=T^{N}\left(T\left(x_{2}\right)\right)=g\left(x_{2}\right)$

We now rewrite the sequence $x_{1}, x_{2}, x_{3}, \cdots$ as

$x_{1}, x_{2}, x_{3}, \cdots, x_{N}, g\left(x_{1}\right) g\left(x_{2}\right) g\left(x_{3}\right) ; \cdot g\left(x_{N}\right) g\left(g\left(x_{1}\right)\right) g\left(g\left(x_{2}\right)\right) g\left(g\left(x_{3}\right)\right) ; \cdots$

This is actually a combination of the $N$ sequences

$$
\begin{aligned}
& x_{1}, g\left(x_{1}\right), g\left(g\left(x_{1}\right)\right), \cdots \\
& x_{2}, g\left(x_{2}\right), g(g(x)), \cdots \\
& x_{3}, g\left(x_{3}\right), g\left(g\left(x_{3}\right)\right), \cdots \\
& \vdots \\
& x_{N}, g\left(x_{N}\right), g\left(g\left(x_{N}\right)\right), \cdots
\end{aligned}
$$

Each row in the above 'array' is obtained by starting at some point of $X$ and iterating with the contraction of $g$. By Theorem 1.1, any such sequence converges to a unique fixed point of $g=T^{N}$, namely $w$. Since each row in the above 'array' is a subsequence of the combined sequence $x_{1}, x_{2}, x_{3}, \cdots$ converging to $w$, the sequence must also converge to, as desired $\diamond$.

\section{Main results}

Let $F$ be a real valued function on a nonempty subset $D$ of the Euclidean space $R^{2}$. A real valued function $\phi$ on an interval $I$ is said to be a solution of the differential equation

$$
d x / d t=F(x, t)
$$

on the interval $I$ if and only if $(\phi(t), t) \in D$ for all $t \in I$, is differentiable on $I$ and $\phi^{\prime}(t)=F(\phi(t) f) \forall t \in I$. 
Bishnu P. Dhungana/Contraction Mapping Principle

Definition 3.1 : Let $F$ be a contriousus real valued finction on a nonempty subset $D$ of the Euclide an space $R^{2}$. A real valued function $\phi_{0}$ an interval I containing $c$ is said to be a solution of the irtegral equation

$$
x(t)=x_{0}+\int_{c}^{t} F(\phi(s), s) d s \quad(t \in I)
$$

if and only if $(\phi(t), t) \in D$ for all $t \in I$, is contimuous on $I$ andfor all $t \in I$

$$
\phi(t)=x_{0}+\int_{c}^{t} F(\phi(s), s) d s \text {. }
$$

The integal $\int_{c}^{t} F(\phi(s), s) d s$ is defined for each $t \in I$ since the function $s \rightarrow F(\phi(s), s)$ is cortimous on I. It is easy to see that the differential equation (3.1) with the initial condition $x(c)=x_{0}$ is equivalent to the integal equation (3.2).

Theoren 3.1: Let $F: R^{\times}[a, b] \rightarrow R$ beafintion of two woriables such that $F(x, t)$ is difined for all $x \in R$ and $t \in[a, b]$. Assum e that $F$ is continuous and that there exists a real nupwber I with

$$
|F(x, t)-F(y, t)| \leq L|x-y|
$$

for all $x, y \in R$ and. Then the differential equation

$$
d x i d t=F(x, t)(3.3)
$$

subject to aninitial condition of the tpee $x(\alpha)=\beta$, has a urique solution

Proof: Lat $X=([a b]$ Then $X i s$ a complete metric spre with the metric

$$
d(x, y)=\sup _{a \leq s}|x(t)-y(t)| .
$$

Define $T: X \rightarrow X$ by

$$
(T(x))(t)=\beta+\int_{a}^{t} F(x(s), s) d s \text {. }
$$

Then the fixedpoints of $T$ are the soltions ofthe irtegral equation on $[a, b]$ and herce these sre the solutions to the differential equation (3.3). To prove the theorem it suficices to show that $T$ has a mique fixed poirt. We finst show that some iterate of $T$ is a contraction of $X$ into itself. To each $x, y \in C[a, b]$ and $t \in[a, b]$, and, we have

$$
\begin{aligned}
& \text { | (T) }(x), A-(T(x), a) \mid
\end{aligned}
$$

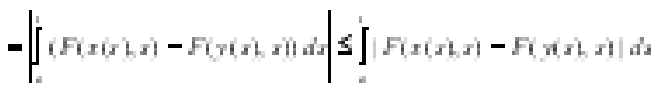

$$
\begin{aligned}
& \left.\leq L \int|x(x)-y(x)| d x=L(t)-a\right) d(x, y) \\
& \leq L \int|(T(x))(s)-(T(y))(s)| \omega \leq L d(x, y) \int_{a}^{c}(s-d) d s \\
& -\frac{E(t-d)^{2} d\left(x_{x} y\right)}{2}
\end{aligned}
$$

By induction, it is easy to see

that $\mid\left(T^{N}(x)\right)(t)-\left(T^{N}(x)\right)(t) \leq \frac{L^{N}(t-a)^{N} d(x, y)}{N !}$.

It then fo llows that

$$
\begin{gathered}
\left.d\left(\Gamma^{N}(x), \Gamma^{N}(y)\right)=\sup (s \leq) \mid\left(\Gamma^{N}(x)\right)(t)-T^{N}(y)\right)(t) \mid \\
\leq \frac{L^{N} d(x, y) \sup _{a \leq s,}(t-a)^{N}}{N !} \\
=\frac{L^{N}(b-a)^{N} d(x, y)}{M !}
\end{gathered}
$$

Maim: $\frac{[L(b-a)]^{N}}{N !} \rightarrow 0 \leq N \rightarrow \infty$

Proof of the daim: Let $A$ be the smallest positive integer such that $L(b-a) \leq A$. Then

$$
\frac{[L(b-a)]^{N}}{I N !} \leq \frac{A^{N}}{I N !}
$$


Butfor $N>A$,

$$
\begin{aligned}
& \frac{A^{A}}{N !}=\frac{A^{A} A^{A-A}}{A !(A+1)(A+2) \cdots(A+(N-A-1))(A+(N-A))} \\
& =\frac{A^{A}}{A !} \frac{A}{A+1} \frac{A}{A+2} \cdots \frac{A}{N-1} \frac{A}{N}
\end{aligned}
$$

and since the product $\frac{A}{A+1} \frac{A}{A+2} \cdots \frac{A}{N-1}<1$, we have

$$
\frac{A^{N}}{N !}<\frac{A^{+1}}{A N N}
$$

Let $\varepsilon>0$ be antinry. Choose $N=\frac{1}{a}\left(\frac{A^{t+1}}{A}+1\right)$. Then $\frac{A^{+1}}{A}-N a-1<N a \leq N \leq \quad \nabla N \geq N$

Thus

$\frac{A^{H+1}}{A N N}<\varepsilon \quad \nabla N \geq N$

From (36), (3.7) and (3.8), we have $\frac{[L(b-a)]^{N}}{N !}<\varepsilon \quad \forall N \geq N$. Thous

$$
\frac{[L(b-a)]^{N}}{N !} \rightarrow 0 \propto N \rightarrow \infty
$$

Clearly there exists a positive integer $M$ such that $\frac{[L(b-a)]^{M}}{M !}<1$ and hence from (3.5), we hare $d\left(T^{M}(x), T^{M}(y)\right) \leq \frac{[L(b-a)]^{M}}{M !} d(x, y)$.

It proves that $T^{M}$ is a contraction of $X$ into itself. By Theorm 2.2, The andique fixed poirt, $s y \phi(t)$ and which is the unique solution to the differentialequation (33). 4

Inpractice, finding whether there is such an I as in Theorem 3.1 is amajor question. This can be seen from the following theorem and some examples.
Theorem 3.2: Let $F: R \times[a, b] \rightarrow R$ be afuntion of twowriable such that $F(x, t)$ is dined for all $x \in R$ and $t \in[a, b]$. Assunethat $F$ is cortinuous, that $F$ is partially differentiable with respect to $x$ and that $\partial F / \partial x$ is boundidt hroughout $R \times[a, b]$. Thenthe differentialequation $d x / d t=F(x, t)$ susject to an initial condition of the type $x(\alpha)=$, has a urique solution

Proof: Assume that $|\partial F / \partial x| \leq L$ for all $x \in R$ and $\in[a, b]$. Fix $t$ and define afinution $G$ of $x$ alone by $G(x)=F(x, t)$. Clearlyis differentiable and by mean whe theorem forin $R$

$$
G(x)-G(y)=G(z)(x-y)
$$

for some $z \in(x, y)$. Hence

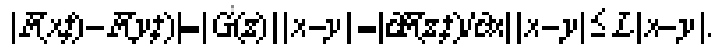

Theproof is completeby Thoorem 3.1. 4

Example 3.1: Let $F(x, t)=t /\left(2^{t}+x^{2}\right)$ for $x \in R$ and $\in[-10,10]$. Then the differential equation $d x / d t=F(x, t) \quad$ with some initial condition $x(\alpha)=\beta$ has a urique solution on $[-10,10]$. For, let $p$ be ary positive real ramber and $x$ ary real number. Then

$$
\begin{aligned}
& P^{-1}-\left[2 x /\left(P^{2}+x^{2}\right)\right]=(P-x)^{2} /\left[P\left(P^{2}+x^{2}\right)\right] \geq 0, \\
& P^{-1}+\left[2 x /\left(P^{2}+x^{2}\right)\right]=(P+x)^{2} /\left[P\left(P^{2}+x^{2}\right)\right] \geq 0 \\
& \text { whichyield }\left|2 x /\left(P^{2}+x^{2}\right)\right| \leq P^{-1} \text { so thet }
\end{aligned}
$$

$\left|2 x\left\|\left(P^{2}+x^{2}\right)^{2}|\leq| 2 x\right\|\left(P^{2}+x^{2}\right)\right| F^{-2} \leq P^{-1}$. (3.10) Using (3.10) and $\partial F(x, t) / \partial x=-2 t x /\left(2^{t}+x^{2}\right)^{2}$, we have

$|\partial F / \partial x|=|t|\left|2 x,\left(2+2^{2}\right)^{2}\right|$

$\leq|t| \mid 2 x /\left(\left(2^{r / 2}+x^{2}\right)^{2}|\leq| t \mid\left[2^{r / 2}\right]^{-3} \leq 10 \times 2^{1 s}\right.$. Since the same canchusion can be dramn for any interval $[-n, n]$ the given differential equation has a mique solution $x(t)$ definted for $t \in R$ 
Bishnu P. Dhungana/Contraction Mapping Principle...

Example 3.2: Let $[a, b]$ be an interval contained in $(0, \infty)$. Let $F(x, t)=1 /\left(t+e^{x}\right)$ for $x \in \boldsymbol{R}$ and $t \in[a, b]$. Then the differential equation $d x / d t=F(x, t)$ with $x(\alpha)=\beta$ has a unique solution on $[a, b]$. For, $|\partial F / \partial x|=e^{x} /\left(t+e^{x}\right)^{2} \leq 1 /\left(t+e^{x}\right) \leq a^{-1}$.

\section{References}

Rubinstein, I. and L. Rubinstein 1998. Partial differential equations in classical mathematical physics. Cambridge University Press, United Kingdom. $675 \mathrm{pp}$.

Bailey, D.F. 1966. Some theorems on contraction mappings Journal of London Mathematical Society 41 101-106.

Yosida, K. 1978. Functional analysis. Springer, Berlin.

Braun, M 1993. Differential equations and theirapplications, Springer-Verlag, New York. 578 pp. 
Nepal Journal of Science and Technology 10 (2009) 